عزل وتثخيص بعض انواع الفطريات من حالات التهاب قناة الاذن الخارجية مع فحص حساسية العزلات لبعض انواع المضادات الفطرية

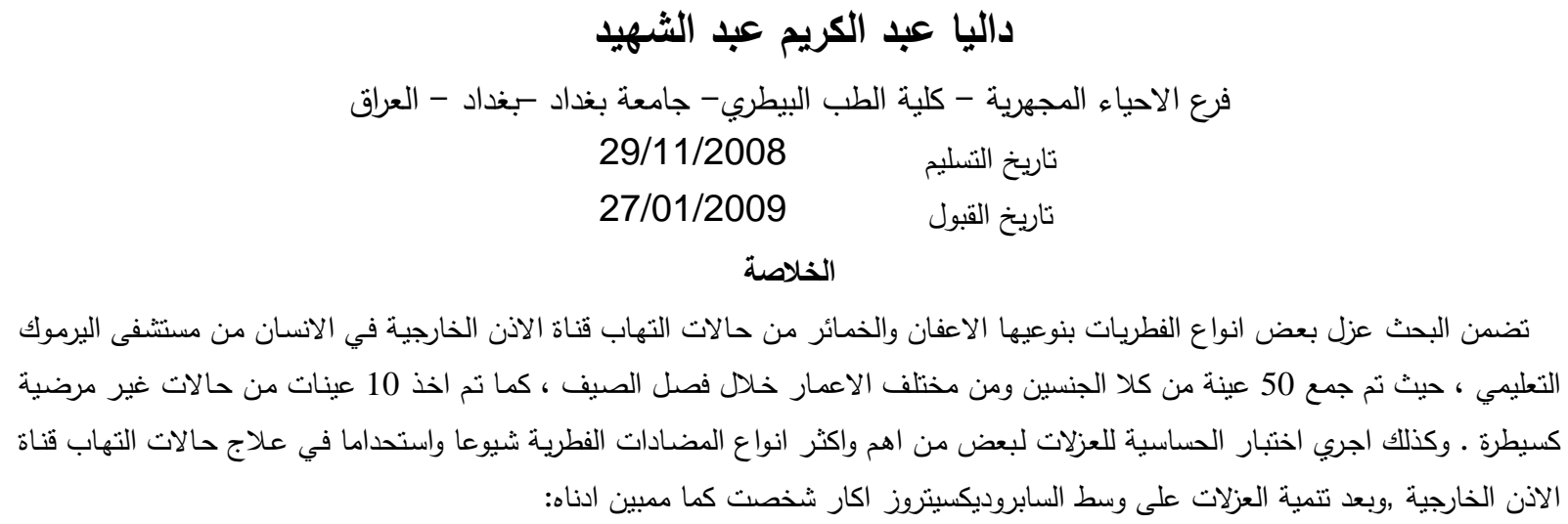

\title{
Summary Isolation and diagnosis of some fungal types from external ear canal infection cases with sensitivity test to some antifungal agents
}

\author{
Dalya abulkareem abdulshaheed \\ Dept. of microbiology - college of Vet. Med.- Baghdad university - Baghdad - Iraq
}

\begin{abstract}
Summary
This research included isolation of some types of fungi of molds and yeasts from cases of inflammation of external ear canal in human from AL- yarmouk Teaching Hospital, by collection of 50 samples from males and females and from different ages during summer season, also 10 samples from non infected cases were taken as control. Also sensitivity test of some isolates against some important antifungal agents which commonly used in the treatments of infection of external ear canale cases.

After cultivation of isolates on saboroud dextrose agar, diagnosed as follows :

9 isolates of Aspergillus niger, 6 isolates of Aspergillus fumigatus, 3 isolates of Aspergillus flavus, one isolate of Aspergillus terrus, 10 isolates of Candida albicans, 5 isolates of Candida tropicalis, 2 isolates of Candida parapsilosis.
\end{abstract}




\section{المجلة الطبية البيطرية العراقية، المجلا 33، العدد 1، السنة 2009}

The results of the sensitivity tests of some isolates against the antifungal agents appeared as follows: the isolates of Aspergillus spp. were very sensitive to Clotrimazole and Econazole, while were less sensitive against Nystatine.

Whereas the species of Candida were very sensitive to Nystatine and Econazole, but less sensitive against Clotrimazole.

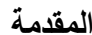

تعتبر الاذن احد الاجزاء المهمـة والحساسـة في الجسم ، حيث تتكون من الصيوان وقناة الاذن الخارجية والاذن الوسطى منتهية بطبلة الاذن وتغطى قناة الاذن الخارجية بالجلد والذي يحتوي على الثعر والغدد الدهنية ، وتقوم الغدد بافراز مادة لزجة ، كما ان الجلد الميت يختلط مع افراز

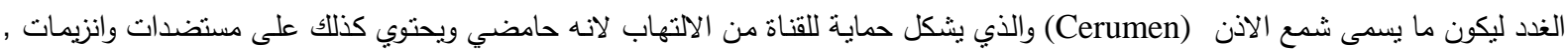
لذا فان اي عامل يؤدي الى تحطيم هذه الطبقة او تقليل حموضتها يؤدي الى التهاب القناة, ومنها ازالة الثمع بالتنظيف الزائد باستخدام أعواد القطن

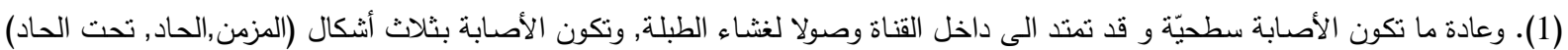
اعتمادا على شدة الأصابة،وقد قنّم (1,2) التهاب قناة الأذن الخارجية الفطري الى ثناثلث أنواع:

1. الالتهاب الفطري الغير مرضي (Non pathogenic mycosis)

حيث عزلت انواع من عفن .Aspergillus spp من المناطق العميقة الرطبة للقناة، كما لوحظت خيوط العفن نامية على شمع الاذن وبقايا الخلايا الظهارية الميتة . الخاع.

2.

3.

$$
\text { حيث لوحظ ان النوعين الاخيرين هما الاكثر حدوثا . }
$$

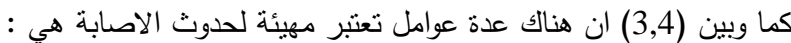

الاصـابة المزمنة للقناة الخارجية للاذن مـع طول فترة استخدام المضـادات الحياتية والمركبات الستيرويدية للاذن وبشكل قطرات ، حدوث اصـابة فطرية خاصه ببعض انواع الخمائر في بعض اعضاء الجسم، مرض السكري، امراض اخرى تغير من الاستجابة المناعية للجسم.

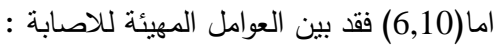

غياب شمع الاذن الرطوبة العالية، زيادة درجات الحرارة،الكلدم الموضعي للاذن (Local truma) ،استخدام المسحات القطنية(عيدان تتظيف الاذن) او قراصـات الثعر ،كثرة السباحة او التعرض للماء يزيل طبقة الثمع ويؤدي الى جفاف القناة السمعية، الاصـابة بالاكزما ، الجيوب الانفية التحسي (asthma) (allergic rhinitis) والرتخدام المضادات الحيانية لفترات طويلة. وعزلت انواع من خميرة .Candida spp من قناة الاذن الخارجية عند النساء في نفس الوقت كانوا مصابين بالالتهاب المهبلي وكذلك بعضهم مصاب بالالتهاب الاظافر الفطري المزمن (5,6). كما وعزلت انواع من عفن .Aspergillus spp من قناة الاذن الخارجية من خـال ملاحظة بقايا الجلد الميت مصبوغة باللون الاسود او الاصفر بينما لوحظت افرازات كريمية بيضاء في حالة الاصابة بخميرة . Candida spp (7), واكدت من من الكثير من البحوث من ان الاصابة تزداد في المناخ الحار اكثر وكذلك لاى السباحين الرياضين ، حيث لوحظ ان من بين كل 8 حالات التهاب قناة

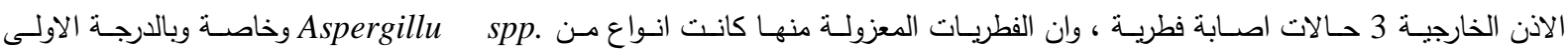
Aspergillus niger

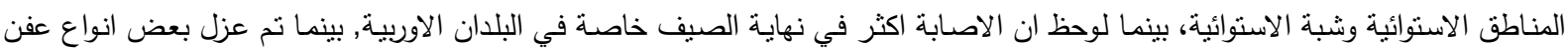
من Candida spp. وخميرة Aspergillus spp. المتناولين للعلاجات المثبطة للمناعة ( $1,8,11$ ) ولاهمية هذا الموضوع فقد هدف هذا البحث الى عزل وتتـخيص بعض انواع الفطريات المسبية لمرض التهاب قناة الاذن الخارجية الفطري لانتشاره عند الاطفال والمراهقين خاصة في فصل الصيف وكذلك اجراء فحص الحساسية للعزلات لغرض اختيار المضاد المناسب للعلاج. 
* جــع النمـاذج : تم جمـع 50 عينة من حالات التهاب الاذن الخارجية في الانسان وبمختلف الاعمـار ومن كلا الجنسين وبهيئة مسحات قطنيـة بالتعاون مع مجموعة من اخصائين انف واذن وحنجرة في مسنتفى اليرموك التعليمي خلال الفترة المتدة ما بين شهر اذار وحتى شهر اب من عام 2008, كما نم جمع 10 عينات اخرى من حالات غير مرضية كنماذج سبطرة. * عزل الفطريات: زرعت العينات على وسط السابرود ديكستروز اكار SDA وبمعدل طبقين لكل نموذج وحضنت الاطباق بدرجة 37 مُ و 25 م ولمدة اسبوع مع ملاحظة النمو كل يومين وفي حالة عدم ظهور النموخلال المدة اعلاه تركت لاسبوع اخر فعند عدم ملاحظة النمو اعتبرت النتيجة سالبة ركما تم عمل مسحة من كل نموذج على شريحة زجاجية مع اضافة هيدروكسيد البوتاسيوم 10 \% وباستخدام صبغة اللاكتوفينول الازرق لغرض وند الفحص المجهري.

\section{Diagnostic test : الفحوص التشخيصية}

- الفحص العياني (Macroscopic test) : فحصت المستعمرات الفطرية من حيث شكلها لونها,قطرها. - الفحص المجهري(Microscopic) : فحصت مجهريا باخذ جزء صغير من المستعمرة ووضعت على شريحة زجاجية وصبغت بصبغة اللاكتوفينول

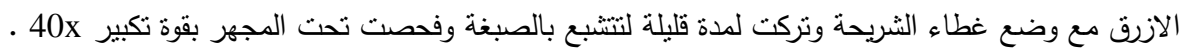

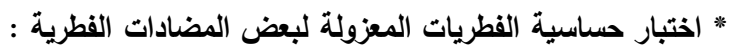

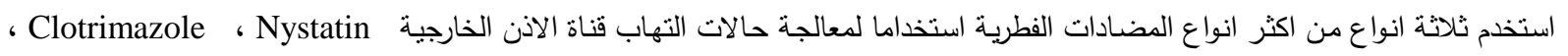
Econazole وبعد تحضير المحاليل القياسية لها مزجت مع الوسط المستخدم وبثلاث تراكيز 0.25 \% 1, 3, 3 \% لكل مضاد فطري وحسب طريقة

$$
\text { اظهرت نتائج البحث عزل مجموعة من الاعفان والخمائر وكما موضح في الجدول (جدول 1). }
$$

\begin{tabular}{|c|c|c|}
\hline نسبة الاصابة\% & عدد العزلات الموجبة & نوع العزلة \\
\hline 25 & 9 & Aspergillus niger \\
\hline 16.66 & 6 & Aspergillus fumigatus \\
\hline 8.33 & 3 & Aspergillus flavus \\
\hline 2.77 & 1 & Aspergillus terrus \\
\hline 27.77 & 10 & Candida albicans \\
\hline 13.88 & 5 & Candida tropicalis \\
\hline 5.55 & 2 & Candida parapsilosis \\
\hline 100 & 36 & المجموع \\
\hline
\end{tabular}

جدول ( 1 ): نوع وعدد الفطريات المعزولة من حالات التهاب قناة الاذن الخارجية في الانسان

كما واظهرت النتائج ان ارتفاع درجات الحرارة والرطوبة اثرت بشكل واضح على زيادة معدلات الاصابة, وتبين ان اكثر نسبة للاصابة كانت عند

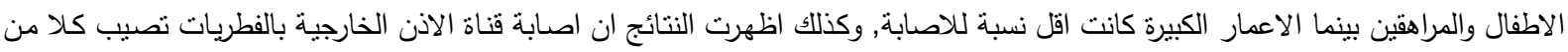
الاناث والذكور الا ان نسبة اصابة الذكور اكثر من الاناث (الجدول 2). 
المجلة الطبية البيطرية العراقية، المجلا 33، العدد 1، السنة 2009

جدول (2): الفئات العمرية الاكثر اصابة مع نسبة الاصابة المئوية للاكور والاناث وتاريخ الحالة المرضية

\begin{tabular}{|c|c|c|c|c|c|}
\hline \multirow[t]{2}{*}{ تاريخ الحالة المرضية } & \multicolumn{2}{|c|}{ الاناث } & \multicolumn{2}{|c|}{ الذكور } & \multirow{2}{*}{ لفئات العمرية } \\
\hline & نسبة الاصابة & الموجد النماذج & نسبة الاصابة & الموبدة النماذج & \\
\hline \multirow{2}{*}{ الالاذنياد للمسابح مع استخدام عيدان تتظيف } & 20 & 2 & 26.92 & 7 & $10-1$ \\
\hline & 50 & 5 & 34.61 & 9 & $20-11$ \\
\hline اصابة جهازية بالفطريات & - & - & 3.84 & 1 & $30-21$ \\
\hline اصلابة بمرض السكري واصابة مهبلية & 20 & 2 & 7.69 & 2 & $40-31$ \\
\hline \multirow{3}{*}{ 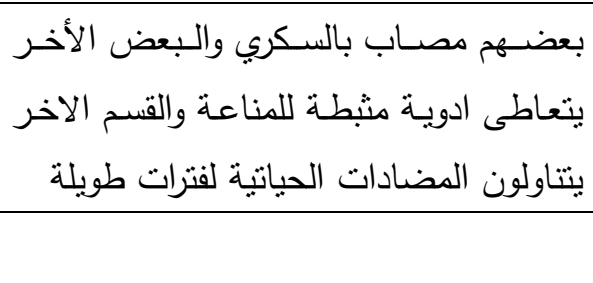 } & 10 & 1 & 19.23 & 5 & $50-41$ \\
\hline & - & - & 7.69 & 2 & $60-51$ \\
\hline & 100 & 10 & 100 & 26 & المجموع \\
\hline
\end{tabular}

"نتائج فحص الحساسية للمضادات الفطرية:

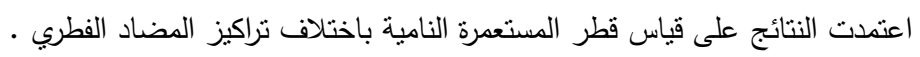

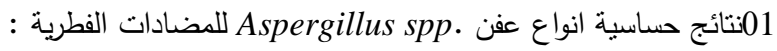

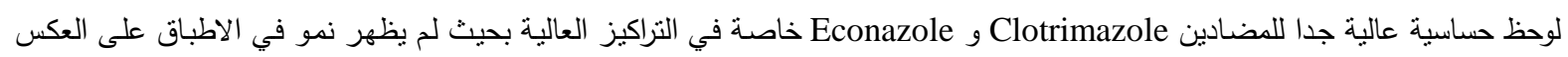
من قلة حساسية للمضاد الفطري Nystatin فقد كان اقل حساسية ( الجدول 3 ).

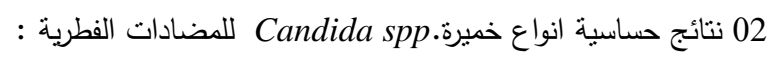

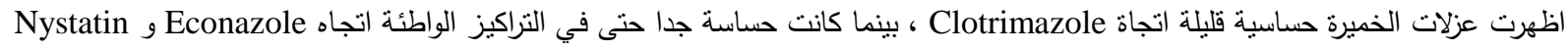

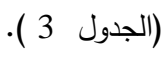


جدول (3): اقطار المستعمرات ب(ملم) بعد اضافة المضادات الفطرية الثلاثة المستعملة للوسط الزرعي بثلاث تراكيز مختلفة

\begin{tabular}{|c|c|c|c|c|c|c|c|c|c|c|}
\hline \multirow{2}{*}{ اطباق سيطرة } & \multicolumn{3}{|c|}{ Nystatin تراكيز } & \multicolumn{3}{|c|}{ تراكيز Clotrimazole } & \multicolumn{3}{|c|}{ Econazole تراكيز } & \multirow[t]{2}{*}{ 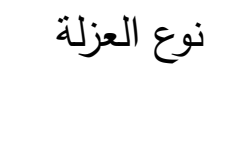 } \\
\hline & $\% 3$ & $\% 1$ & $\% 0.25$ & $\% 3$ & $\% 1$ & $\% 0.25$ & $\% 3$ & $\% 1$ & $\% 0.25$ & \\
\hline 45 & 27 & 36 & 40 & - & 2 & 21 & - & 7 & 20 & $\begin{array}{c}\text { Aspergillus } \\
\text { niger }\end{array}$ \\
\hline 49 & 29 & 38 & 43 & - & 4 & 24 & - & 5 & 22 & $\begin{array}{l}\text { Aspergillus } \\
\text { fumigatus }\end{array}$ \\
\hline 42 & 23 & 31 & 38 & - & 3 & 15 & - & 6 & 19 & $\begin{array}{c}\text { Aspergillus } \\
\text { flavus }\end{array}$ \\
\hline 47 & 26 & 34 & 41 & - & 6 & 17 & - & 7 & 19 & $\begin{array}{c}\text { Aspergillus } \\
\text { terrus }\end{array}$ \\
\hline 3 & - & - & 1 & 0.8 & 2 & 2.7 & - & 1 & 2.1 & $\begin{array}{l}\text { Candida } \\
\text { albicans }\end{array}$ \\
\hline 3.5 & - & - & 0.5 & 1 & 1.8 & 2.5 & - & 1.4 & 2 & $\begin{array}{c}\text { Candida } \\
\text { tropicalis }\end{array}$ \\
\hline 2.5 & - & - & 0.8 & 0.5 & 1.5 & 2 & - & 1 & 1.7 & $\begin{array}{c}\text { Candida } \\
\text { parapsilosis }\end{array}$ \\
\hline
\end{tabular}

المناقشة

يعتبر التهاب قناة الاذن الخارجية الفطري من الامراض الثائعة الحدوث، فقد لوحظ زيادة الاصـابة بالمرض في موسم الصيف وذلك لرغبة الناس

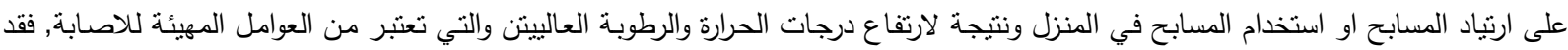

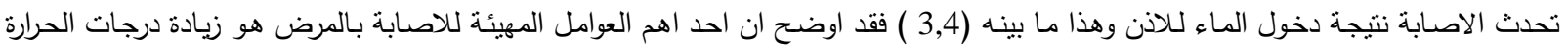

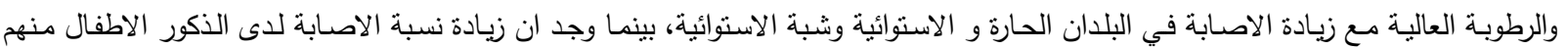
والمراهقين عنه في الاناث وذلك لرغبة الذكور في هذة الاعمار على السباحة اكثر من الاناث خاصة في مجتمعانتا ، فيما كانت الاعمار الاكثر سنا

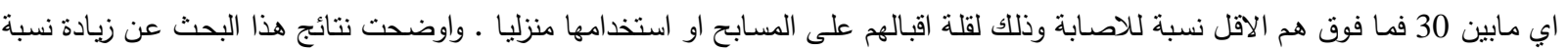
الاصابة لدى الاثخاص المصابين بمرض السكري وكذللك الاشخاص المتناولين لعلاجات تؤدي لاضطرابات مناعية وهذامابينة (1,4) الذي اكد ان

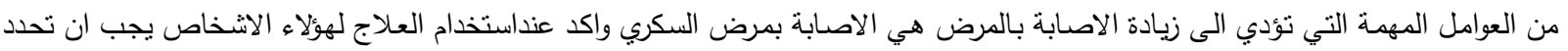
نسبة السكر في الدم لمعرفة اختيار العـلاج المناسب لمثل هذا الالتهاب ، واوضـح (2,14) ان المصابين بامراض مناعية او المتتاولين لعلاجات

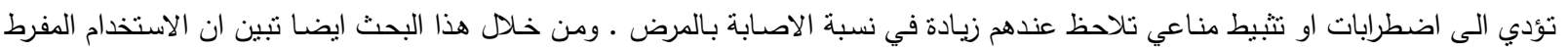

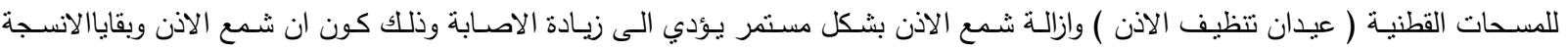
الظهارية الميتة لجلد قناة الاذن الخارجية يعتبران حماية لها من الاصـابة بالفطريات او الجراثيم الاخرى مما يؤدي الى تغير في الاس الهيدروجيني

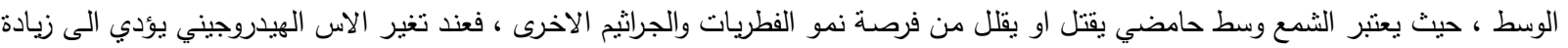
احتمالية نمو الفطريات اكثر (3,6 ،كذلك الاصابة بالامراض الجلدية مثل الاكزما او الاصـابة بالتهاب الجيوب الانفية التحسسي وكذلك الكدم تؤدي التئي جميعها لزيادة نسبة الاصـابة وهذا مابينة واوضحة (1,10) الذي اعتبر هذه الاصـابات اعلاه من العوامل التي تساعد في حدوث وزيادة الاصسابة 
الفطرية لقناة الاذن الخارجية، واوضحت الدراسة ايضا ان زيادة نسبة الاصـابة تكون واضحة اكثر لاى الاشخاص المتعاطين للمركبات الاستيرودية وللمضـادات الحياتيـة ولفترات طويلة وقد بين (2,9) من ان استخدام هذه العلاجات لفترات زمنية طويلـة تؤدي لزيادة الاصـابة الفطريـة لقناة الاذن

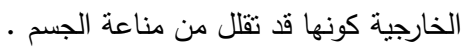

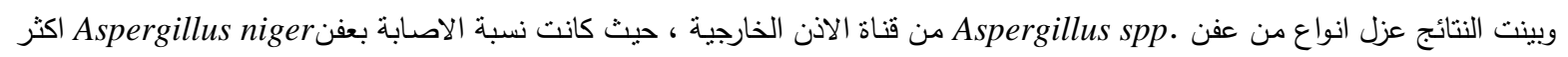

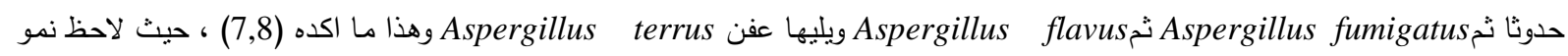
الخيوط الفطرية على او بين شمع الاذن وكذلك على الجلد المبطن لقناة الاذن الخارجية .

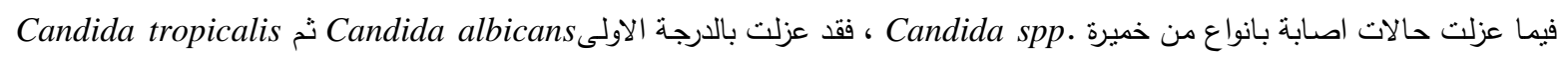
ثم Candida parapsilosis وهذا مـا يؤكد (4,5) ، حيث عزل بعض انواع من هذه الخميرة من حالات التهاب قناة الاذن الخارجيـة منها Candida ، Candida kefyr ،Candida famata ، Candida parapsilosis ، Candida tropicali ،Candida albicans و guilliermondii

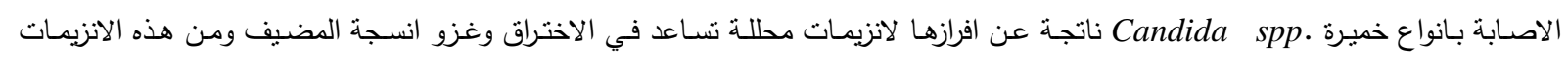

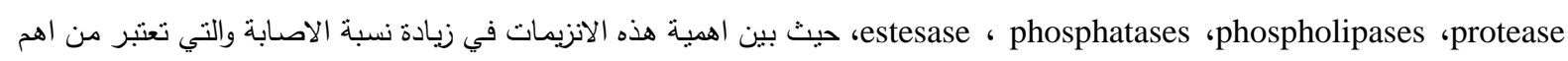
عوامل الضراوة لدى انواع هذه الخميرة ، ولهذا السبب كانت الاصدابة والاعراض شديدة لدى الاثخاص المصابين بها واقل شدة لدى المصـابين . Aspergillus spp. بفطر

فيما يخص اختبار الحساسية فقد استخدمت ثلاثة من اهم انواع المضادات الفطرية والاكثر شيوعا في علاج حالات التهاب قناة الاذن ، فقد كان المضاد الفطري Econazole هو الاكثر تاثيرا على فطر .Candida spp. وختى في في التراكيز الواطئة وذلك لتركيبه الكيمياوي كونـه ينتمي لمجموعة Azoles التي تعمل على تتبيط سـايتوكروم الخلية الفطرية ويثبط الانزيم المسؤول عن تصنيع الاركوستيرول

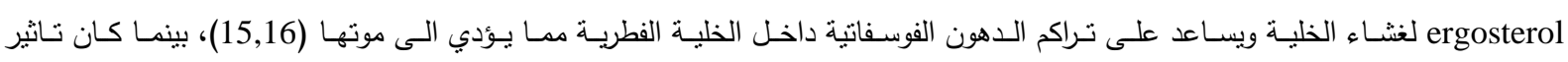
Candida spp. ويعود سبب هذا الاختلاف في التتبيط الى قلة التركيز المطلوب لنقليل نمو وقتل الخميرة بالرغم من كونه ينتمي لمجموعة Azoles (16)، اما تأثنير Candida فقد كان واضحا على خميرة

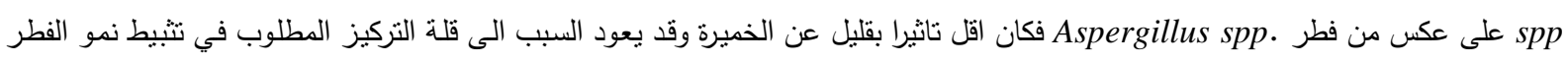
على الرغم من كون هذه المضاد يرنبط مع الاركوستيرول ergosterol لغشاء الخلية الفطرية مما يؤدي الى تغير النفوذية وتسريب محتويات الخلية

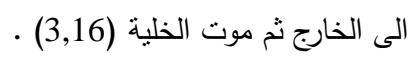

1- Kaur, R.; N. Mittal; M. Kakkar; A. K. Aggarwal and M. D. Mathur. (2005). Otomycosis : A clinicomycologic study Ear Nose Throat J. 79: 606 -9.

2- 2-Chander J; Maini S and Subrahmanyan S.( 2004). Otomycosis clinicmycological study and efficacy of mercurochrome in its treatment.Mycopathologia; 35:9 - 12.

3- Garry JP. (2007). Otitis externa. Clinics in Dermatology. 15:22-25.

4- Irina and Vennewald. ( 2008). otomycosis. Clinics in Dermatology. 23:565- 571.

5- Ghannum MA and Abu Elteen KH. (2007). Pathogenicity determinants of Candida. Mycoses; 33: $265-82$.

6- Amigot SL; Gomez CR;Luque AG and Ebner G. (2003). Microbiological study of external otitis in Rosario City, Argentina.Mycoses; 46: 312-5

7- Supiyaphun P;Sampatanukul P and Sukumalpaiboon P.( 2007). Aspergillus Colonization in external ear canal. Otolaryngol Head Neck Surg;125:281 - 2.

8- Bellini C;Antonini P and Ermanni S.(2007). Otitis externa due to Aspergillus spp. Scand J Infect Dis.;35:284-8.

9- Rowlands S; Devalia H and Smith Cl . (2007). Otitis externa in UK . Br J Gen Pract. Jul;51(468):533-8.

10- Vennewald I; Schfnlebe J and Klemm E. (2003). Mycological and histological investigations in humans with external ear infections. Mycoses; 46:

12- 8 . 
11- Stern; J. C.; M. K. Shah; and F.E. Lucente.( 2001). In vitro effectiveness of 13 agents in otomycosis and review of the literature. Laryngoscope. 98:1173-7.

012الجبوري ، سيناء وليد (2000) دراسـة خمج الفطريات الانتقالية في الردهات الباطنية في مستشفى اليرموك التعليمي - رسالة ماجسنير في

$$
\text { الاحباء المجهرية -كلية الطب - الجامعة المستتصرية. }
$$

13- Macdonald F and Odds FC. . (2004). Purified Candida albicans proteinase in the serological diagnosis of systemic candidosis; 243: $2409-11$.

14- Valentina A.;Nenad A. and Aleksandar D .( 2008 ).Protease activities of Candida spp. Isolated from immunocompeten patients with otomycosis.;23:171-174.

15- Ho T; Vrabec JT and Yoo Dl. ( 2006). Otomycosis: clinical features and treatment implications. Otolaryngol Head Neck Surg. Nov;135(5):787-91.

16- Bassiouny A.; T. Kamel; M. K. Moawad and D. S. Hindawy.( 2007). Broad spectrum antifungal agents in otomycosis. J Laryngol Otol. 100:867-73. 